\title{
PROKLA-Redaktion
}

\section{Editorial: Ökonomie der Flucht und der Migration}

Bis zum Frühsommer 2015 kamen die meisten Menschen, die Krieg, Verfolgung, Staatszerfall und wirtschaftlicher Not in ihren Heimatländern entfliehen und Europa erreichen wollten, aus Somalia und dem Sudan sowie aus Syrien und Afghanistan. In der Regel versuchten sie über das Mittelmeer nach Italien oder Malta zu gelangen, viele davon bezahlten dies mit ihrem Leben. So standen im Jahr 2014 den mehr als 200.000 Flüchtlingen, die die europäische Küste erreichten, mehr als 3.000 gegenüber, die im Meer ertrunken waren. Während Papst Franziskus Lampedusa besuchte, um dort die allgemeine Gleichgültigkeit gegenüber dem Leiden der Flüchtlinge anzuprangern, und während die italienische Marine mit der Operation Mare Nostrum insgesamt mehr als 100.000 Flüchtende in Seenot rettete, fiel führenden PolitikerInnen der EU nicht mehr dazu ein, als diese Maßnahmen der italienischen Regierung als Einladung zur Flucht zu kritisieren. An Hilfen dachten andere Länder als Italien nicht und eine finanzielle Beteiligung der EU daran wurde abgelehnt. Eine Änderung der Modalitäten für die Einwanderung und die Beantragung von Asyl wurde nicht einmal erwogen. Stattdessen wurde im Herbst 2014 für die EU-Grenzagentur Frontex die Operation Triton beschlossen, bei der Schiffe, Flugzeuge und Hubschrauber die EU-Außengrenzen sichern sollten. Diese Linie setzte sich im Mai 2015 mit dem
Beschluss zur Bekämpfung des „Schlepperunwesens" im Mittelmeer konsequent fort und offiziell wurde verkündet: „Nach dem Vorbild des Anti-Piraterie-Einsatzes vor der somalischen Küste sollen EUKriegsschiffe Schlepperboote zerstören, die zur Überfahrt von Flüchtlingen über das Mittelmeer genutzt werden." (Demling/ Wittrock 2015) In Somalia war Anfang der 1990er Jahre jegliche funktionierende Staatlichkeit zusammengebrochen, was europäische (ebenso wie außer-europäische) Fischfangflotten zu massiver illegaler Fischerei nutzten, womit wiederum den Einheimischen weitgehend die Lebensgrundlage entzogen wurde, weshalb sich in der Folge viele von ihnen als Ausweg der Piraterie zuwandten. Dies bewog die EU 2008, wie Heide Gerstenberger in PROKLA 163 analysiert (Gerstenberger 2011), zur Militärmission Atalanta. Wie sein Vorgänger ist der neue Plan rechtlich höchst umstritten, auch lag die Vermutung nahe, man wolle dabei nicht Flüchtlinge vor kriminellen Schleppern, sondern die europäischen Länder vor den Flüchtlingen schützen. Es ist bisher unklar, ob und wieweit dieses Vorhaben umgesetzt wurde und was es bewirkte, dennoch folgte ihm im Februar 2016 der Beschluss der NATO, einen Marineverband in die Ägäis zu schicken, um abermals „Schleuserbanden“ zu bekämpfen.

Inzwischen hat sich die Situation allerdings grundlegend gewandelt. Allein 
in der ersten Jahreshälfte 2015 erreichten mehr als 100.000 Flüchtlinge über Griechenland und die sogenannte 'Balkanroute' Österreich und Deutschland. Die Regierungen dieser Länder hatten bisher gemeint, sie bräuchten sich dank der für sie günstigen Dublin-Regelungen (wonach das EU-Land, das Flüchtlinge als erstes betraten, für deren Asylverfahren zuständig war) weder mit den Gründen der Flucht noch mit dem Schicksal der Flüchtlinge selbst weiter beschäftigen und könnten dies getrost den Staaten an den südlichen EU-Außengrenzen überlassen. Daran konnten auch die sich verstärkt seit 2012 selbst organisierenden Refugees in Deutschland - eine Bewegung, die von Andrea Plöger in PROKLA 177 untersucht wird (Plöger 2014) - nichts ändern, selbst wenn es kurzzeitig so aussah, als könnten die Refugee-Proteste in Hamburg die Dublin-Regelung möglicherweise kippen. Nun stehen die Flüchtlinge mit einem Mal vor der eigenen Haustür und es stellt sich mit neuer Dringlichkeit die Frage, wie sie auf die einzelnen EU-Länder verteilt werden sollen. Die Fluchtrouten haben sich seither, insbesondere nach der faktischen Schließung der 'Balkanroute' im März 2016, erneut verschoben und sie werden dies weiterhin tun, da die Abschottung Europas die Menschen nicht davon abhalten wird, sich weiterhin auf den - wenn auch noch so gefährlichen - Weg zu machen. Die Zahl der Flüchtlinge, insbesondere aus Syrien, ist mittlerweile um ein Vielfaches angewachsen, wobei es für SyrerInnen immer schwieriger geworden ist, das Land zu verlassen. Auf höchster EU-Ebene blieben das Denken in Begriffen von Abwehr und Grenzkontrollen sowie das ungebrochene Vertrauen in den Erfolg militärischer Operationen allerdings unverändert.

War es der deutschen Regierung in der Griechenland-Krise noch gelungen, ihre speziellen Vorstellungen von Bankenrettungen durch EU-Mittel in Kombination mit einer rigiden Austeritätspolitik gegenüber sämtlichen EU-Partnerländern - selbst gegenüber denjenigen, die sich in einer ähnlichen Lage wie Griechenland befanden durchzudrücken, so fiel dieses trügerische Bild einer einigen und harmonischen EU nun wie ein Kartenhaus zusammen. Dies wird nicht zuletzt an der katastrophalen Situation in Griechenland sowie in den Lagern an der griechisch-mazedonischen Grenze, der damit einhergehenden fortschreitenden Destabilisierung dieser ungewollten Auffangregion und der hilflosen Passivität der obersten EU-Gremien offenbar. In mehreren Ländern ließen sich konservative und sozialdemokratische Regierungen von einem erstarkenden rechten Lager vor sich hertreiben. Dessen traditionelle Slogans in der Art von "La France aux français“ (Jean-Marie Le Pen 1984) oder „Österreich zuerst“ (Jörg Haider 1992) wurden wieder zunehmend populär, sodass führende PolitikerInnen meinten, sie müssten den Partnerländern gegenüber mit „unzumutbaren Belastungen" argumentieren und jegliche Verteilungsquoten innerhalb der EU entweder überhaupt ablehnen (Lettland, Litauen, Slowakei, Ungarn) oder zumindest niedrig halten: Die österreichische Regierung hat für 2016 eigenmächtig eine "Obergrenze“ festgelegt, die polnische erklärt, sie wolle nur "christliche" Flüchtlinge aufnehmen. Am radikalsten trat hierbei Viktor Orbán in Ungarn auf, der im Februar 2016 eine Volksabstimmung ankündigte, von der er sich Rückhalt für seine ausgrenzende Politik erhofft: „Wir Ungarn meinen, dass Verteilungsquoten für Flüchtlinge und Migranten ohne den Rückhalt des Volkes Machtmissbrauch gleichkommen." (Abendblatt 2016)

In Deutschland, dem Land, das bisher innerhalb der EU die meisten Flüchtlinge aufgenommen hat, lässt sich ebenfalls ein 
Rechtsruck verzeichnen. Mit dem Einzug der AfD in mehrere Landtage - trotz (oder wegen?) der Forderung nach einem Schießbefehl (Beatrix von Storch) - bestätigen die jüngsten Wahlergebnisse diesen Trend nun endgültig auch in den Parlamenten. Auf der Regierungsebene zeigte sich längere Zeit ein eher gespaltenes Bild. Die Bundeskanzlerin hat - aus Gründen, die einige mit ihrem protestantischen Hintergrund erklären, andere als zukunftsorientierte politische Option sehen - ihre bisherige Linie der Nicht-Befassung mit dem Thema Flüchtlingspolitik im letzten Jahr aufgegeben, und es zumindest bis Anfang 2016 immer wieder abgelehnt, Obergrenzen für den Zuzug von Flüchtlingen festzulegen, womit sie in weiten Teilen ihrer eigenen Partei wie insbesondere in der SchwesterPartei CSU auf heftige Kritik gestoßen ist. Parallel dazu wurden im Rahmen des Asylpakets II jedoch Verschärfungen des Asylrechts durchgesetzt, welche zuletzt erkämpfte Errungenschaften wie die Abschaffung der Residenzpflicht wieder zurücknehmen, den Familiennachzug aussetzen und die Abschiebung traumatisierter Menschen ermöglichen. Weitere Verschlechterungen sind auf dem Weg. Nach dem jüngst mit der Türkei geschlossenen und bereits in Kraft getretenen Abkommen, welches von allen EU-Staaten ratifiziert wurde, sind Geflüchtete, die auf illegalem Weg aus der Türkei nach Griechenland einreisen, nach einem Schnellverfahren dorthin wieder abzuschieben: „Im Gegenzug möchten die EU-Staaten für jeden syrischen Abgeschobenen einen syrischen Flüchtling aus der Türkei auf legalen Wegen aufnehmen“- Pro Asyl zufolge nicht nur ein „bizarrer Plan“, sondern vor allem eine Aushebelung der Genfer Flüchtlingskonvention (Pro Asyl 2016). Bestandteil dieser Vereinbarung ist, dass die Türkei Geld und die Aussicht auf eine
Lockerung der Visa-Pflicht für TürkInnen im Rahmen der EU-Beitrittsverhandlungen erhält. Gleichzeitig setzt sich die EUPolitik der Abgrenzung nach Außen nun auch mehr und mehr innerhalb Europas gegen die übrigen EU-Länder durch. Fünfundzwanzig Jahre nachdem man hier das Fallen von Mauern und Grenzzäunen bejubelt hat, werden allerorten neue errichtet.

Wenn es um die Gründe dafür geht, dass Menschen unter schwierigsten Umständen bereit sind, ihre Heimat aufzugeben, unterscheiden PolitikerInnen und Mainstream-Medien meist Kriege und schlechte wirtschaftliche Verhältnisse: erstere gelten als grundsätzlich legitim, letztere hingegen nicht, da den Flüchtlingen unterstellt wird, sie wollten einfach nur ein „besseres Leben“. Dabei wird ausgeblendet, dass diese beiden Gründe in der Realität meist gar nicht zu trennen sind. Wenn Menschen keine Möglichkeiten mehr sehen, ihr Überleben zu sichern, ist dies oftmals eine Folge von früheren oder andauernden Kriegen, der politischen Zerrüttung von Staaten - oder auch der Zerstörung bislang einigermaßen funktionierender Subsistenzwirtschaften und lokaler Märkte, weil diese ausländischem Kapital oder Exporten aus wirtschaftlich überlegenen Ländern geöffnet wurden (wie bei dem genannten Beispiel der Fischerei in Somalia). Die Regierungspolitik mehrerer EU-Länder oder der EU insgesamt hat viele der krisenhaften Umstände mitproduziert: durch politische Interventionen, durch Waffenlieferungen oder durch wirtschaftliche Abkommen zur Öffnung von Märkten. Zu den im engeren Sinn ökonomischen Fluchtursachen gehört, dass Kapital aus EU-Ländern (wie aus anderen Weltgegenden) seit längerer Zeit auf der Suche nach neuen lukrativen Anlagefeldern ist und darin durch politische Weichenstellungen unterstützt wurde, die 
häufig neokoloniale Züge tragen, so unter anderem seit den 1960er Jahren durch bilaterale Investitionsabkommen (BITS) oder die Gemeinsame Agrarpolitik der EU. Die offizielle Rhetorik, die Öffnung der afrikanischen Märkte für ausländische Investitionen würde Beschäftigung und Wachstum, Wohlstand und „Partnerschaften unter Gleichberechtigten " fördern, wird mittlerweile durch umfangreiche Erfahrungen widerlegt. Der Abbau von Rohstoffressourcen schafft allenfalls schlechte Arbeitsplätze, dafür aber gleichzeitig massive Umweltzerstörungen, und ist zudem mit einem hohen $\mathrm{Maß}$ an Korruption verbunden, an der keineswegs nur die lokalen Eliten, sondern stets ebenfalls ausländische Investorengruppen beteiligt sind („It takes two to tango"). Dazu kommt in neuerer Zeit in vielen Ländern massives landgrabbing, das sich nicht nur auf Grund und Boden, sondern häufig auch auf Meeresressourcen erstreckt, was die Existenzgrundlage von Bäuerinnen und Bauern, von Fischerinnen und Fischern zerstört und sie zu Flucht oder Abwanderung zwingt. Sogenannte Partnerschaftsabkommen erlauben den EU-Fangflotten den Zugang zu afrikanischen Gewässern gegen unzureichende Ausgleichszahlungen. Sie tragen zur Zerstörung der einheimischen Fischerei bei und verschlechtern in diesen Ländern zudem häufig die Versorgung mit Fisch, der ein wichtiges Nahrungsmittel darstellt. Ähnlich sieht es mit anderen landwirtschaftlichen Bereichen aus, wenn hochsubventionierte EU-Produkte in afrikanische Länder exportiert werden. In einer ZEIT-Reportage vom Dezember 2015 wurde anschaulich geschildert, wie Agrarkonzerne aus Süditalien die Märkte in Ghana mit billigem Tomatenmark überschwemmen, gegen das einheimische Kleinproduzenten nicht konkurrieren können, daher gezwungen sind, ihre Höfe aufzugeben, in Ghana kaum andere Beschäftigung finden und das Land verlassen. Das traurige Ende des Liedes ist, dass viele der Geflohenen unter schlechtesten Bedingungen als Erntehelfer in Italien arbeiten: „Dort pflücken sie jene Tomaten, die später in ihre alte Heimat in Afrika exportiert werden und die Existenz der heimischen Bauern bedrohen." (Kraupa/ Lobenstein 2015: 23) Diese Hintergründe kommen in der gegenwärtigen Debatte zur Flüchtlingspolitik nur selten zur Sprache.

Exportiert werden jedoch nicht nur Lebensmittel, sondern auch Waffen, die etwa beim „Exportweltmeister" Deutschland - zwar nur einen minimalen Anteil aller Exporte ausmachen (in den letzten Jahren ein Prozent oder weniger), für die Länder, in die geliefert werden, oder in denen sie letztlich landen, aber verheerende Folgen haben. So hat die seit Jahrzehnten praktizierte politische wie militärische Unterstützung Saudi-Arabiens durch den Westen dazu geführt, dass der angebliche "Stabilitätsanker" nicht nur selbst militärisch hochgerüstet ist, sondern darüber hinaus als Drehscheibe des Waffenhandels für die gesamte Region fungiert. Die Waffen werden aus Deutschland und aus anderen Ländern geliefert, weil die Regelungen für Rüstungsexporte entweder ohnehin schwach ausfallen oder, wie im Fall von Deutschland, die Kontrolle äußerst lax gehandhabt wird. An ihren Bestimmungsorten heizen sie Kriege aller Art an und erschweren Verhandlungslösungen, tragen somit ebenso dazu bei, dass Menschen ihr Heil nur noch in Auswanderung oder Flucht sehen. Die geringe volkswirtschaftliche Bedeutung dieser Exporte zeigt, wie leicht sie verzichtbar wären, würden Regierungen die Wünsche der Rüstungsfirmen nicht stets allzu bereitwillig erfüllen. In ihrem Beitrag über deutsche Waffenlieferungen in den Irak, nach Afghanistan und 
Syrien untersucht Dorothea Schmidt diese Zusammenhänge.

Doch nicht nur Krieg und produziertes Elend führen zu neuen Migrationsbewegungen, sondern oftmals gerade die wirtschaftliche und politische Stabilisierung von Ländern und Regionen. So ist in vielen afrikanischen Städten in den vergangenen Jahrzehnten eine neue Mittelschicht entstanden, die nach Mobilität strebt und die globalen Verkehrs- und Kommunikationsmittel nutzt, genau wie die Mittelschichten auf anderen Kontinenten. Hier offenbart sich ein grundlegendes Missverständnis in der öffentlichen und politischen Debatte um die Rolle der 'Hilfe vor Ort'. Zwar mögen sich politische und wirtschaftliche Fluchtursachen durch internationale 'Entwicklungszusammenarbeit' möglicherweise eindämmen lassen. Diese Hilfe bildet aus linksliberaler Perspektive daher eine logische Alternative - oder vielleicht eher eine Ergänzung - zur 'Festung Europa' der Rechten. Doch führt der wirtschaftliche Aufstieg und die politische Stabilisierung zerrütteter Länder nicht unbedingt zu weniger, sondern häufig lediglich zu anderen Formen der Migration. „Wenn es vor allem Elend wäre, das Migrationen auslöst, warum wandern dann hunderte Millionen völlig verarmter Menschen nicht aus Ländern der Dritten Welt aus?", wie Albert Kraler und Christof Parnreiter in PROKLA 140 pointiert fragten (Kraler/ Parnreiter 2005: 327). Es gilt also politisch anzuerkennen, dass Mobilität und Migration in einer globalisierten Welt ganz einfach Tatsachen sind. Diese können gestaltet, aber nicht verhindert werden. Das Rad der Geschichte lässt sich auch auf diesem Feld nicht zurückdrehen.

Wenn es um die Flucht selbst geht: um die Maßnahmen zur Abwehr von Flüchtlingen und um die schließliche Aufnahme von denjenigen, die es dennoch geschafft haben, dann werden wirtschaftliche Motive einzig und allein bei den Schleppern gesehen, von denen man annimmt, dass sie ein illegales und einträgliches Geschäft betreiben (und bei dem sie, wie tatsächlich mehrfach geschehen, oftmals das Leben ihrer "Kundschaft“ aufs Spiel setzen). Aber Flüchtlinge können genauso gut für Unternehmen, die im Rahmen der Legalität operieren, zu einem Geschäft werden. Und schließlich stellt sich die Frage, inwieweit sie auf den Arbeitsmärkten in den EULändern integriert oder ausgegrenzt werden, da ihnen dort häufig nur illegale und/ oder informelle Beschäftigungsformen offen stehen. Auch diesen oftmals wenig beleuchteten Aspekten widmet sich das vorliegende Heft.

Bei der Ökonomie von Prozessen der Flucht und Migration handelt es sich um eine Vielzahl unterschiedlicher Konstellationen, die vom Menschenhandel bis zur ehrenamtlich organisierten Fluchthilfe reichen und staatliche Organe ebenso wie organisierte Kriminalität umfassen. Vom Menschenhandel sind häufig Frauen und Kinder betroffen, die zwangsweise in andere Länder verbracht werden, um sie dort der Prostitution, der Arbeitsausbeutung oder dem Organhandel auszuliefern. Dagegen sind Flüchtlinge Menschen, die aus eigenem (wenn auch nicht freiem) Entschluss ihr Heimatland verlassen. Während vor 1990 Flüchtlinge aus Ostblockländern im Westen zumeist mit Verständnis und Unterstützung rechnen konnten und die Fluchthelfer als „Helden" galten, hat es seither rhetorisch wie politisch einen grundlegenden Wandel gegeben. Am Beispiel von Marokko, dessen See- und Landgrenzen zu Spanien unter Mithilfe der EU aufgerüstet und militarisiert wurden, zeigt Angela Schweizer, wie Geflüchtete, die aus afrikanischen Ländern zunächst nach Marokko migrieren, aber 
eigentlich nach Europa weiterziehen wollen, in diesem „Zwischenland“ stranden, in hauptsächlich informelle oder kriminelle Beschäftigungen abgedrängt werden und sich mitunter selbst im Schlepperwesen verdingen.

ArbeitsmigrantInnen früherer Jahre sahen sich in vielen europäischen Ländern immer schon mit heftigen Ressentiments und kultureller Abwertung konfrontiert, wobei es bereits vor 1990 immer rigidere Beschränkungen für die Zuwanderung gab. Insbesondere die deutsche Regierung vertrat stets, Deutschland sei kein „Einwanderungsland“ und entlarvte damit den Mythos des „aufgeklärten“ und „fortschrittlichen“ Europa als Hort von Freiheit und Menschenrechten. Globalisierung wurde seither immer eindeutiger als Freiheit des Kapitals zur Überschreitung aller Grenzen verstanden, während die globalen Wanderungen von Menschen einzudämmen waren. Dieser Diskurs wird von zunehmend populären rassistischen Konstruktionen von Bürgerschaft begleitet, die homogene Nationen beschwören und an frühere Kernelemente kolonialen Denkens anknüpfen. Auch die Rede von den 'Opfern' der 'Schlepperbanden' hilft den betroffenen MigrantInnen wenig. „Eine eigene Entscheidung darüber, wo und wie sie leben möchten, wird ihnen auf diese Weise abgesprochen. Stattdessen werden sie medial zu Opfern der organisierten Kriminalität reduziert. Am Ende erscheinen Polizei-Razzien auf diese Weise als humanitäre Geste gegenüber Migranten, die faktisch jedoch deren Abschiebung und die Verschärfung von Grenzkontrollen und Einreisebestimmungen zur Folge haben." (Euskirchen u.a.2009:77) Die Grenzlinien zwischen Europa und seinem „Außen“ bestehen somit nicht nur entlang der Mittelmeerküste, sondern ebenso in den Köpfen. Politischen Ausdruck findet der Wandel darin, dass europäische Staaten gesetzlich festgeschriebene Menschenrechte immer häufiger zugunsten von polizeilichen und militärischen Abwehrmaßnahmen in den Hintergrund drängen. Sie sehen sich daher letztlich weder für den Transport noch für die Unterbringung von Flüchtlingen verantwortlich, sondern delegieren diese Aufgaben teils gewollt, teils ungewollt an Schleuser in informellen Netzwerken, an profitorientierte Unternehmen im formalen Sektor, an eingesessene NGOs oder an neue ehrenamtliche Initiativen. Gerade die Unterbringung von Geflüchteten in den euphemistisch als 'Gemeinschaftsunterkünfte' bezeichneten Lagern und umfunktionierten Gebäuden hat sich vor diesem Hintergrund zu einem lukrativen Geschäftsfeld entwickelt. In Berlin etwa erhalten Hostels für die Unterbringung von Flüchtlingen derzeit bis zu 50 Euro pro Nacht. Wenn die Stadt in Zahlungsverzug gerät, werden die ausgegebenen Gutscheine allerdings mitunter nicht mehr angenommen - und die Asylsuchenden bleiben dann ohne Dach über dem Kopf. Die Schweizer Firma ORS Service GmbH erwirtschaftet mit dem Betrieb des zentralen österreichischen Aufnahmelagers in Traiskirchen einen Gewinn von einer Million Euro pro Jahr. In zwei Beiträgen wird thematisiert, welche Probleme und Ambivalenzen sich daher durch die verschiedenen Akteursgruppen ergeben. Sebastian Muy untersucht die Bedingungen sozialer Arbeit in Sammelunterkünften gewerblicher Träger in Berlin und zeigt auf, wie widersprüchlich sich die Rolle von SozialarbeiterInnen dort gestaltet, da diese sich häufig in einem Spannungsfeld von zum Teil gegenläufigen Interessen der Beteiligten aufreiben: zwischen den eigenen professionellen Ansprüchen, der staatlichen Gewährleistungspflicht und der Gewinnorientierung privater Betreiber. Silke van Dyk 
und Elène Misbach stellen demgegenüber die zahlreichen Initiativen zum Empfang und zur Unterstützung von Geflüchteten in den Zusammenhang einer allgemeinen Restrukturierung (wohlfahrts-)staatlicher Politik, bei der die gezielte Indienstnahme der „Ressource Gemeinschaft“ in Form von Ehrenamt, Freiwilligenarbeit, Nachbarschaftshilfe und zivilgesellschaftlichem Engagement zum einen den Abbau sozialer Leistungen kompensieren und auffangen soll, zum anderen aber auch ein Einfallstor für Prozesse der De-Professionalisierung, De-Standardisierung und Prekarisierung von Arbeit im Sozialbereich darstellen kann.

Schließlich ist zu fragen, warum Flüchtlinge es in den europäischen Ankunftsländern so schwer haben, auskömmliche Erwerbsmöglichkeiten zu finden. Zunächst könnte man erwarten, dass das Kapital in diesen Ländern ein großes Interesse an Arbeitskräften hat, die oftmals fachliche Kompetenzen und geringe Erwartungen an gute Bezahlung mitbringen, also die industrielle Reservearmee vergrößern und einen weiteren Druck auf die Senkung des Lohnniveaus ausüben könnten. Und in der Tat wurde die 'Flüchtlingskrise' in Deutschland von interessierten Kreisen sogleich genutzt, um den gerade eingeführten Mindestlohn von 8,50 Euro zu torpedieren. Die Kompromissformel zwischen CDU und SPD läuft nun darauf hinaus, die Praktikumszeiten für Asylberechtigte und anerkannte Flüchtlinge, bei denen vom Mindestlohn abgewichen werden kann, auf mindestens sechs Monate zu verlängern. Zugleich beschwören viele Unternehmen und ihre Verbände seit Längerem eine zu befürchtende „demografische Katastrophe“ (kritisch hierzu PROKLA 146/2007), da die Erwerbsbevölkerung überaltert sei und junger Nachwuchs fehle. Doch selbst Regierungen, die nicht von rechten Populisten dominiert werden, machen sich deren alarmistische Argumente der drohenden "Überfremdung“ mehr und mehr zu eigen und setzen in ihrer Flüchtlingspolitik auf Abschottung und Ausgrenzung. Vereinzelt forderten Unternehmensverbände auch eine schnellere Anerkennung von ausländischen Bildungsabschlüssen, aber insgesamt blieb das Interesse an der Integration der Flüchtlinge in die heimischen Arbeitsmärkte lange Zeit gering, wie u.a. an den jeweiligen Ausbildungs- und Arbeitsverboten zu sehen war. Neuerdings scheint sich hier, wie Karin Scherschel in ihrem Beitrag ausführt, ein Paradigmenwechsel anzukündigen, da Flüchtlinge nunmehr schnelleren Zugang zum Arbeitsmarkt bekommen sollen, was sich in neuen rechtlichen Regelungen niederschlägt. Allerdings ist dies vor dem Hintergrund zu sehen, dass ihnen dabei in der Mehrzahl aller Fälle lediglich prekäre Beschäftigungen offenstehen und diese zwiespältige Integration im Rahmen des nach wie vor praktizierten rigiden Kontrollregimes der „aktivierenden Arbeitsmarktpolitik" stattfindet. Dazu kommt, dass sie bei ihrer Arbeitssuche in Konkurrenz zu einer Vielzahl von Migrantinnen und Migranten stehen, die aus EU-Ländern mit hoher (Jugend-)Arbeitslosigkeit kommen. Die Bandbreite ist groß: Es kann sich beispielsweise um Roma aus Rumänien handeln, die in Deutschland als Scheinselbstständige arbeiten, oder auch um spanische, griechische oder portugiesische Jung-AkademikerInnen, die der horrenden Arbeitslosigkeit in ihren Altersgruppen zu entfliehen suchen - letztlich eine Folge der anhaltenden Austeritätspolitik, die den Krisenländern von der EU aufgezwungen wurde. Etwas anders liegt der Fall, wenn etwa in der spanischen Landwirtschaft Saisonarbeitskräfte aus Marokko oder subsaharischen Ländern rekrutiert werden, 
da diese zirkuläre Migration den dortigen Arbeitgebern sehr viel attraktiver als die dauerhafte Beschäftigung von Flüchtlingen erscheint. Hier erweist sich eine neue „Gastarbeiter“-Politik als Mittel, um das Reservoir an „billigen und willigen“ Arbeitskräften zu vergrößern.

Fabian Georgi unternimmt schließlich den Versuch, einen theoretischen Rahmen für materialistische Analysen von Migration, Flucht und Migrationspolitik zu schaffen. Er schließt damit an regimetheoretische Ansätze an, die sich seit den frühen 2000er Jahren im Anschluss an die Debatte um die 'Autonomie der Migration' entwickelt haben - siehe dazu etwa den Beitrag von Martina Benz und Helen Schwenken in PROKLA 140 (Benz/ Schwenken 2005). Doch während diese vor allem auf die agency der migrantischen Akteure abstellen und auf die diskurs- und praxistheoretische Ebene fokussieren, schlägt Georgi vor, die strukturtheoretischen Momente stärker zu betonen und die kritische Grenzregimeforschung politökonomisch zu fundieren. „[D]ie Bedingungen emanzipatorischer Veränderungen lassen sich ohne grundlegende politökonomische Analysen nicht angemessen verstehen “, so Georgi in diesem Heft.

Außerhalb des Schwerpunkts greift Alex Demirović mit Religion eines der Themen des letzten Heftes auf, plädiert für eine Politik der radikalen Immanenz und verteidigt die Gegenwart gegen die Hoffnung auf ein besseres Morgen. Die Moderne gehe mit einer Zukunftsorientierung einher, die zugleich die Lösungen für kommende Zeiten verspricht - ohne sie je einzulösen. Das komme einer Verewigung von Herrschaftsverhältnissen gleich. Demirović fordert deshalb in seinem Text mit Walter Benjamins Begriff der „Jetztzeit" eine Neuorientierung von (auch linker) Politik auf die Gegenwart.
Felix Syrovatka legt in seinem Einspruch die gesellschaftlichen und ökonomischen Hintergründe der jüngsten Protestbewegung in Frankreich offen: Nuit Debout. Auch wenn die Bewegung, die die Nacht zum Tag macht, hoffnungsvoll stimmt, so Syrovatka, bedarf es einiger Veränderungen und Voraussetzungen, dass daraus eine wirklich gesellschaftsveränderte Kraft entsteht, die die gegenwärtigen Reformvorhaben noch stoppen kann und einen Politikwechsel einleitet.

Während in den letzten beiden PROKLA-Ausgaben Dario Azzellini ${ }^{1}$ und Mario Candeias die Möglichkeiten und Grenzen linker (Partei-)Politik in Südeuropa, vor allem Spanien und Griechenland, kritisch und im Dissens diskutierten, beschäftigt sich Aaron Tauss mit der Krise des Chavismus in Venezuela. Gerne diskutiert die Linke politische Projekte und neue Akteure, wenn sie Veränderungen versprechen, scheut sich aber oft davor, Rechenschaft darüber abzulegen, wenn sich die auch noch so vorsichtigen Erwartungen zerschlagen - das gilt für die Politik Syrizas in Griechenland genauso wie für die linken Regierungen in Lateinamerika. Wir hoffen, die Debatte in den kommenden Ausgaben fortsetzen zu können.

\section{Dorothea Schmidt und Sandra Sieron}

(für die Redaktion)

Ende April 2016 fand die Mitgliederversammlung der Vereinigung zur Kritik der politischen Ökonomie e.V. statt, der

1 An dieser Stelle entschuldigen wir uns dafür, dass wir in der letzten Ausgabe den Namen von Dario Azzellini konsequent falsch geschrieben haben. 
Verein, der die PROKLA herausgibt und die Redaktion und den Redaktionsbeirat wählt. Katharina Hajek verstärkt zukünftig die Redaktion, Sandra Sieron bereits seit letztem Jahr. Michael Heinrich wechselt von der PROKLA-Redaktion in den Beirat, den Sabine Nuss bedauerlicherweise verlässt. Wir danken an dieser Stelle für die langjährige Mitarbeit und Unterstützung.

\section{Literatur}

Abendblatt (2016): Verteilungsquoten für Flüchtlinge: Ungarn will das Volk befragen. URL: abendblatt.de (24.2.2016), Zugriff: 26.3.2016.

Benz, Martina/Schwenken, Helen (2005): Jenseits von Autonomie und Kontrolle: Migration als eigensinnige Praxis. In: PROKLA 35(3): 363-378.

Demling, Alexander/Wittrock, Philipp (2015): Flüchtlingskatastrophe im Mittelmeer:
Europa ringt um die Rettung. URL: spiegel. de (20.4.2015), Zugriff: 23.3.2016.

Euskirchen, Markus u.a. (2009): Wie Illegale gemacht werden. Das neue EU-Grenzregime. In: Blätter für deutsche und internationale Politik 54(7): 72-80.

Gerstenberger, Heide (2011): Atalanta: Militär gegen Piraterie. In: PROKLA 41(3): 317-334.

Kraler, Albert/Parnreiter, Christof (2005):

Migration Theoretisieren. In: PROKLA 35(3): 327-344.

Kraupa, Mathias/Lobenstein, Caterina (2015):

Ein Mann pflückt gegen Europa. In: DIE ZEIT, Nr. 51.

Plöger, Andrea (2014): Die Proteste der Refugees. Eine Bewegung von den Rändern ins Zentrum Europas. In: PROKLA 44(4): 585-600.

Pro Asyl (2016): Warum der Deal mit der Türkei eine Schande für Europa ist. URL: proasyl.de (18.3.2016), Zugriff: 26.3.2016.

\section{Der PROKLA Förderverein}

Die PROKLA erscheint seit 1971 und bietet politisch engagierte sozialwissenschaftliche und ökonomische Analysen. Allein von den Verkaufserlösen kann sich die PROKLA nicht finanzieren und in die Abhängigkeit von Parteien oder großen Verlagen wollte sie sich nie begeben. Deshalb wird die PROKLA von einem Förderverein herausgegeben, der „Vereinigung zur Kritik der politischen Ökonomie e.V.“, die jährlich in ihrer Vollversammlung die Redaktion der Zeitschrift wählt und die nächsten Themenschwerpunkte diskutiert.

Kritische Sozialwissenschaft kann nicht dem Markt überlassen werden. Ohne solidarische Strukturen und finanzielle Unterstützung sind Zeitschriften wie die PROKLA kaum möglich. Die regelmäßigen finanziellen Beiträge der Vereinsmitglieder ermöglichen das Erscheinen der PROKLA, sie schaffen die Voraussetzungen für Kontinuität und Planbarkeit, wie sie für die Redaktionsarbeit unabdingbar sind. Wir freuen uns über weitere Mitglieder, regelmäßige Spenden oder einmalige Zuwendungen. Weitere Informationen teilen wir gerne per E-Mail mit (redaktion@prokla.de).

Vereinigung zur Kritik der politischen Ökonomie e.V.

IBAN: DE17 100100100538135100 - BIC: PBNKDEFF

Postbank Berlin 